\title{
Velocity-space diffusion of solar wind protons in oblique waves and weak turbulence
}

\author{
E. Marsch and S. Bourouaine \\ Max-Planck-Institut für Sonnensystemforschung, Katlenburg-Lindau, Germany
}

Received: 22 July 2011 - Revised: 27 September 2011 - Accepted: 9 November 2011 - Published: 24 November 2011

\begin{abstract}
The fast solar wind is permeated by all kinds of plasma waves which have a broad range of wavelengths and occur on many different scales. Kinetically, a plasma wave induces ion-wave interactions which can within the quasilinear theory be described as a diffusion process. The impact this diffusion may have on the shape of the proton velocity distribution function (VDF) is studied. We first analyse theoretically some of the possible kinetic effects of the waves on the ions. Then the model predictions are compared with the detailed in-situ plasma measurements made by the Helios spacecraft on 14 April 1976 at $0.3 \mathrm{AU}$ and found to comply favourably with resonant diffusion of protons in obliquely propagating magnetohydrodynamic waves. In particular, the shape at the edges of the VDFs at positive proton velocities in the wind frame can be well explained by cyclotron-resonant diffusion of the protons in oblique fast magnetoacoustic and Alfvén waves propagating away from the Sun.
\end{abstract}

Keywords. Interplanetary physics (MHD waves and turbulence; Plasma waves and turbulence; Solar wind plasma)

\section{Introduction}

The solar wind is accessible to in situ plasma measurements, and thus represents a unique space plasma laboratory to study generally wave-particle interactions. Kinetic processes in the solar wind have been reviewed by Marsch (2006) a couple of years ago. The measured proton velocity distributions in fast solar wind reveal as salient non-thermal features the strongly anisotropic core and field-aligned beam (Marsch et al., 1982). The origin and regulation of these characteristics remain under discussion, but it seems clear that waves play a major role in shaping the observed velocity distribution functions (VFDs). Marsch and Tu (2001a) found the first evidence for the occurrence of diffusion-related plateaus formed by the solar wind protons. They appeared to be in resonance predominantly with parallel Alfvén/ion-cyclotron waves and to undergo continuously pitch-angle scattering. The resulting temperature anisotropy and plateau formation through resonant pitch-angle diffusion of solar wind protons was then studied by Tu and Marsch (2002). Moreover, Bourouaine et al. (2010) could show that the proton anisotropy and the Alfvén/ion-cyclotron-wave intensity were linked and positively correlated.

In this paper, we will corroborate these findings and demonstrate that wave-induced diffusion can significantly shape the observed VDFs, in particular in their tails defined as regions below the $10 \%$ level of the respective maxima. It turns out that oblique propagation is the key property which enables particles at positive as well as negative resonant speed (referred to as $V_{\|}$in the solar wind frame along the local magnetic field) to diffuse effectively along segments of circles centered in the Alfvén velocity. This basic process is mainly driven by low-frequency and obliquely propagating Alfvén/ion-cyclotron waves and by slow/fast magnetosonic waves, of which the Alfvén waves are known to be the major and energetically dominant component of the turbulence in fast solar wind streams (Tu and Marsch, 1995). The slow mode waves (sometimes also interpreted as pressurebalanced structures, PBS, occurring on a wide range of scales (Marsch and Tu, 1993; Kellogg and Horbury, 2005; Yao et al., 2011; Howes et al., 2011)) also contribute, but the oblique fast mode waves seem to be least important (Matthaeus et al., 1991; Marsch and Tu, 1993; Tu and Marsch, 1994).

Correspondence to: E. Marsch

(marsch@mps.mpg.de)

Published by Copernicus Publications on behalf of the European Geosciences Union. 


\section{Quasi-linear diffusion operator}

Before we discuss the physical consequences of the diffusion equation, we reiterate some of the basic equations and definitions needed subsequently. The quasi-linear theory (QLT) has been described in many seminal original articles. Therefore, we can here refer to the textbooks of Melrose and McPhedran (1991) and Stix (1992) for the original literature. In QLT it is assumed that the electromagnetic wave fields can generally be Fourier-decomposed in plane waves with the frequency, $\omega(\boldsymbol{k})$, and growth rate, $\gamma(\boldsymbol{k})$, for any particular wave mode for a given wave vector $\boldsymbol{k}$, which may have any direction with respect to the constant background field, $\boldsymbol{B}_{0}$. We may also require the related wave dispersion relation, which for any linear wave mode in a multi-component plasma has been calculated many times and can be found for instance in the books of Stix (1992) or Brambilla (1998).

The spectral energy density of the magnetic field of a mode is given by $\mathcal{B}(\boldsymbol{k})=|\tilde{\boldsymbol{B}}(\boldsymbol{k})|^{2} /(8 \pi)$ and evolves according to

$$
\frac{\partial}{\partial t} \mathcal{B}(\boldsymbol{k})=2 \gamma(\boldsymbol{k}) \mathcal{B}(\boldsymbol{k})
$$

which follows from the Fourier decomposition of the magnetic field vector as

$$
\boldsymbol{B}(\boldsymbol{x}, t)=\sum_{\boldsymbol{k}} \tilde{\boldsymbol{B}}(\boldsymbol{k}) e^{i \boldsymbol{k} \cdot \boldsymbol{x}} e^{-i \int_{0}^{t} d t^{\prime}\left[\omega\left(\boldsymbol{k}, t^{\prime}\right)+i \gamma\left(\boldsymbol{k}, t^{\prime}\right)\right]},
$$

where $\boldsymbol{x}$ is the spatial coordinate and $t$ is the time, and the Fourier-transform, $\tilde{\boldsymbol{B}}(\boldsymbol{k})$, of the magnetic field vector, $\boldsymbol{B}(\boldsymbol{x})$, are assumed to be defined such that they have the same physical dimension.

The quasi-linear diffusion equation describes the evolution of the velocity distribution function, $f_{j}\left(V_{\|}, V_{\perp}, t\right)$, of any particle species $j$ in an inertial frame of reference, in which the particles and waves are supposed to propagate. We will throughout this paper assume that the VDF is normalized to a density of unity. The general diffusion equation for any type of waves in a magnetized plasma has originally been derived by Kennel and Engelmann (1966). It is calculated in a transparent way in the textbook Stix (1992) and can be written after Marsch and Tu (2001b) and Marsch (2002) concisely in the form

$$
\begin{gathered}
\frac{\partial}{\partial t} f_{j}\left(V_{\|}, V_{\perp}, t\right)=\sum_{s=-\infty}^{+\infty} \sum_{\boldsymbol{k}} \hat{\mathcal{B}}(\boldsymbol{k}) \frac{1}{V_{\perp}} \times \\
\frac{\partial}{\partial \alpha}\left(V_{\perp} v_{j}\left(\boldsymbol{k}, s ; V_{\|}, V_{\perp}\right) \frac{\partial}{\partial \alpha} f_{j}\left(V_{\|}, V_{\perp}, t\right)\right),
\end{gathered}
$$

where the pitch-angle gradient was introduced. It is given by the combined velocity derivative

$$
\frac{\partial}{\partial \alpha}=V_{\perp} \frac{\partial}{\partial V_{\|}}-\left(V_{\|}-\frac{\omega(\boldsymbol{k})}{k_{\|}}\right) \frac{\partial}{\partial V_{\perp}} .
$$

The relevant phase velocity appearing here is given a name of its own as $C(\boldsymbol{k})=\omega(\boldsymbol{k}) / k_{\|}$. The magnetic field fluctua- tion spectrum is normalized to the background-field energy density:

$\hat{\mathcal{B}}(\boldsymbol{k})=\left(\frac{\tilde{B}(\boldsymbol{k})}{B_{0}}\right)^{2}\left(\frac{k_{\|}}{k}\right)^{2} \frac{1}{1-|\hat{\boldsymbol{k}} \cdot \boldsymbol{e}(\boldsymbol{k})|^{2}}$.

The term in the denominator comes from the replacement of the electric field by the magnetic field power density as derived in Marsch (2002). The circular and longitudinal components of the wave-polarization unit vector (see, e.g. Melrose and McPhedran, 1991, or Mann et al., 1997, for multispecies plasma) are here defined as

$e_{ \pm}(\boldsymbol{k})=e_{\mathrm{X}}(\boldsymbol{k}) \pm i e_{\mathrm{y}}(\boldsymbol{k})$ and $e_{\mathrm{Z}}(\boldsymbol{k})$.

Thereby $e_{-}$corresponds to right, respectively $e_{+}$to left hand polarization of the wave. In Eq. (3), it turned out to be physically meaningful to introduce what we may call an ion-wave relaxation or wave-particle collision rate. It is defined as

$$
\begin{gathered}
v_{j}\left(\boldsymbol{k}, s ; V_{\|}, V_{\perp}\right)=\pi \Omega_{j}^{2} \delta\left(\omega(\boldsymbol{k})-s \Omega_{j}-k_{\|} V_{\|}\right) \\
\quad \times\left|\frac{1}{2}\left(J_{s-1} e_{+}+J_{s+1} e_{-}\right)+\frac{V_{j}(\boldsymbol{k}, s)}{V_{\perp}} J_{s} e_{\mathrm{Z}}\right|^{2} .
\end{gathered}
$$

Note that the quantity (Eq. 7) has indeed the dimension of an inverse time or rate, and it can be considered as transition probability (Melrose and McPhedran, 1991) for inelastic wave-particle scattering. Here we also introduced the $s$-order resonance speed,

$$
V_{j}(\boldsymbol{k}, s)=\frac{\omega(\boldsymbol{k})-s \Omega_{j}}{k_{\|}}=C(\boldsymbol{k})-\frac{s}{y_{\|}} V_{\mathrm{A}},
$$

and made use of the Bessel function (with index $s$ ), $J_{s}=$ $J_{s}\left(k_{\perp} V_{\perp} / \Omega_{j}\right)$, with $J_{s}(0)=\delta_{s, 0}$. We defined above a normalized parallel wave vector as $y_{\|}=k_{\|} V_{\mathrm{A}} / \Omega_{j}$.

In the previous equations and in what follows the definitions are as usual: The speed of light is denoted by $c$, the ion charge by $e_{j}$, its density by $n_{j}$, the mass by $m_{j}$, and the plasma frequency of species $j$ is $\omega_{j}^{2}=\left(4 \pi e_{j}^{2} n_{j}\right) / m_{j}$. The ion gyrofrequency carries the sign of the charge and reads $\Omega_{j}=\left(e_{j} B_{0}\right) /\left(m_{j} c\right)$. The mass density of species $j$ is defined as $\rho_{j}=n_{j} m_{j}$, and the total mass density is $\rho=\sum_{j} \rho_{j}$. The Alfvén speed is based on it and thus defined as $V_{\mathrm{A}}^{2}=$ $B_{0}^{2} /(4 \pi \rho)$. The fundamental Eq. (3), with all its ingredients as listed above, is quoted here without derivation as the starting point of our subsequent calculations.

\section{Cyclotron resonance and diffusion plateaus}

It is obvious from the structure of the diffusion operator (3), involving the pitch-angle-gradient derivation (4), that any velocity distribution which is a function of the quantity

$E\left(V_{\|}, V_{\perp}\right)=\frac{1}{2}\left(V_{\perp}^{2}+V_{\|}^{2}\right)-\int_{0}^{V_{\|}} d V_{\|}^{\prime} C\left(V_{\|}^{\prime}\right)$ 
is conserved along the characteristics of Eq. (4). We here assumed that the phase speed is a function of $V_{\|}$, and then formally integrated it with respect to that variable (Isenberg and Lee, 1996). Thus for a dispersion-less wave, $E\left(V_{\|}, V_{\perp}\right)$ is simply the specific kinetic energy of a particle moving in the wave frame of reference, as it was defined previously by the phase speed $C(\boldsymbol{k})$. Therefore, any distribution function $f_{j}=f_{j}(E)$ is a steady-state solution of the diffusion equation.

In addition, the resonance condition must also be obeyed:

$V_{\|}=C\left(y_{\|}, y_{\perp}\right)-\frac{s}{y_{\|}} V_{\mathrm{A}}$.

Here $y_{\perp}=k_{\perp} V_{\mathrm{A}} / \Omega_{j}$, and the phase speed $C$ is assumed to be given by the solution of a particular dispersion relation, of which we will discuss typical examples below. Let us here consider the general case, $C(\boldsymbol{y})=g\left(y_{\|}, y_{\perp}\right) V_{\mathrm{A}}$. Through the resonance condition (10), we may then consider (at a fixed $k_{\perp}$ ) the parallel velocity to be a function of the parallel wave vector, i.e. we have $V_{\|}=V_{\|}\left(y_{\|}\right)$, of which we can calculate the derivative, in order to replace the differential in the integral (9), and thus change the variable of integration (Isenberg and Lee, 1996). We obtain

$\frac{d V_{\|}}{d y_{\|}}=\frac{d C}{d y_{\|}}+V_{\mathrm{A}} \frac{s}{y_{\|}^{2}}=V_{\mathrm{A}}\left(\frac{s}{y_{\|}^{2}}+\frac{d g}{d y_{\|}}\right)$,

and with its help find that the integral in Eq. (9) which we call $I$ is equal to:

$$
\int_{0}^{V_{\|}} d V_{\|}^{\prime} C\left(V_{\|}^{\prime}\right)=V_{\mathrm{A}}^{2} \int_{y_{0}}^{y_{\|}} d y_{\|}^{\prime} g\left(y_{\|}^{\prime}, y_{\perp}\right)\left(\frac{s}{y_{\|}^{\prime 2}}+\frac{d g}{d y_{\|}^{\prime}}\right) \text {. }
$$

For dispersion-less Alfvén waves the situation becomes simple, as $C_{\mathrm{A}}=V_{\mathrm{A}}$, and $V_{\|}=V_{\mathrm{A}}\left(1-s / y_{\|}\right)$, and thus $y_{0}=s$. Also, $g=1$, and the derivative of $g$ vanishes. Then the integration can be readily carried out, and by exploiting the resonance condition yields for Eq. (12) the simple result: $I=V_{\|} V_{\mathrm{A}}$. Then the energy (up to an arbitrary constant) per mass unit becomes

$E_{\mathrm{A}}\left(V_{\|}, V_{\perp}\right)=\frac{1}{2}\left(V_{\perp}^{2}+\left(V_{\|}-V_{\mathrm{A}}\right)^{2}\right)$.

These solution characteristics define the quasilinear diffusion plateaus, which in this case are circles centered at the Alfvén speed, and are defined by the specific kinetic energy of a particle in the wave frame.

For strongly dispersive waves like ion-cyclotron waves, the full dispersion relation is needed, and to solve the integral (12) one may require numerical integration. In the solar wind context, this has been done by Heuer and Marsch (2007) to evaluate the apparent diffusion plateaus in the measured VDFs of solar wind protons, which were first detected by Marsch and Tu (2001a) in the Helios plasma data. Exemplary velocity distributions revealing distinct plateaus can be found in these two papers.

\section{Kinetic Alfvén waves: dispersion relation and impos- sibility of cyclotron resonance}

To discuss another important and non-trivial dispersion relation, we consider the so called kinetic Alfvén wave (KAW), which is a dispersive plasma wave including an electric field along the background magnetic field. The KAW theory has a long history and many applications, in particular recently to the solar wind in the context of gyrokinetic theory (Howes et al., 2008). For a review of this wave in the space plasma context see the paper by Hollweg (1999). The simplified dispersion relation (here for cold electrons, but a more complete treatment is contained in Hollweg's paper) reads:

$\omega=k_{\|} V_{\mathrm{A}} \sqrt{\frac{1+\left(k_{\perp} v_{\mathrm{p}} / \Omega_{\mathrm{p}}\right)^{2}}{1+\left(k_{\perp} c / \omega_{\mathrm{e}}\right)^{2}}}$,

with the ion thermal speed defined as $v_{j}=\sqrt{k_{\mathrm{B}} T_{j} / m_{j}}$, the ion temperature as $T_{j}$, and Boltzmann's constant is $k_{\mathrm{B}}$. Thus we find that the above function $g$ only depends on $k_{\perp}$ and consequently

$C_{\mathrm{KAW}}=V_{\mathrm{A}} g\left(y_{\perp}\right)$.

Note, however, that this dispersion relation has only a limited range of validity, and strictly speaking requires that $\omega \ll \Omega_{j}$. Here we will as a nontrivial example use the KAW and disregard this restriction for the time being. Again like for the Alfvén wave the derivative of $g$ for the KAW is zero, i.e. $g^{\prime}=0$, and thus the integral (12) can be directly evaluated as follows,

$I=g V_{\mathrm{A}}\left(V_{\|}+(1-g) V_{\mathrm{A}}\right)$,

whereby the resonance condition $V_{\|}=V_{\mathrm{A}}\left(g-s / y_{\|}\right)$for a KAW has been exploited. Except for an unimportant constant, we obtain the plateau (energy) equation in the concise form

$E_{\mathrm{KAW}}\left(V_{\|}, V_{\perp}\right)=\frac{1}{2}\left(V_{\perp}^{2}+\left(V_{\|}-C_{\mathrm{KAW}}\right)^{2}\right)$.

Note that according to Eq. (14) $g>1$, and thus the phase speed of the kinetic Alfvén wave, $C_{\mathrm{KAW}}$, is always larger than the Alfvén speed $V_{\mathrm{A}}$.

We recall that Hollweg (1999) found the KAW to be elliptically polarized in its transverse field components as well as compressive, i.e. there also is a longitudinal component, so that all three components of the polarization unit vector $\boldsymbol{e}(\boldsymbol{k})$ are nonzero and contribute to the matrix element (Eq. 7). The lowest-order Bessel functions are about 0.5 for $\beta_{j}=1$ and $y_{\perp} \geq 1$, as considered here, and thus $v_{j} \leq \Omega_{j}$. Therefore, diffusion in the KAW field would, in principle, occur quickly, say within several gyro periods of the ions under consideration, and its effectiveness would mainly depend on the relative spectral intensity (Eq. 5), which determines the strength of the "diffusion constant" at the relevant $\boldsymbol{k}$ in Eq. (3). 
Whereas for $\beta_{j}$ of order unity, the normalized parallel wave number $y_{\|}$can in fact be considerably smaller than one, it has to be near one in low-beta conditions (like in the solar corona) in order to match the resonance condition (10), which for the standard lowest-order cyclotron resonance at $s=1$ of a thermal particle of species $j$, with $V_{\|}=-v_{j}$ propagating oppositely to a KAW wave with phase speed given by Eq. (15), can be written

$V_{\mathrm{A}} / y_{\|}=g V_{\mathrm{A}}+v_{j}$.

But for $g \approx 1$ we have $C_{\mathrm{KAW}} \approx V_{\mathrm{A}}$, and then the KAW slows down and according to Eq. (14) becomes a high-frequency wave, a situation that is inconsistent with the original assumptions made in its derivation (Hollweg, 1999). Therefore, the resonance requirement always forces the corresponding wave frequency to become close to the gyrofrequency, $\omega / \Omega_{j}=y_{\perp} g k_{\|} / k_{\perp} \approx 1$, and for KAW this is not consistent with the original dispersion relation (14). In conclusion, the plateaus calculated formally for KAW in Eq. (17) are not accessible to thermal protons, as they cannot comply with the resonance condition.

It is worth noting that the typical anisotropy of the KAW near the proton gyroscale can also be estimated from the dispersion relation (14) and shown to satisfy the following relation:

$\frac{k_{\perp}}{k_{\|}}=y_{\perp} \frac{g\left(y_{\perp}\right) \Omega_{\mathrm{p}}}{\omega \sqrt{\beta}} \gg 1$,

where, for the inequality, we used the values, $\beta \approx 1, g \approx \sqrt{2}$, $y_{\perp}=1$ and $\omega \approx 0.1 \Omega_{\mathrm{p}}$, for which the anisotropy of the KAW fluctuations is $k_{\perp} / k_{\|} \approx 14$, i.e. they have a propagation angle larger than $85^{\circ}$. However, according to some recent observations (Chen et al., 2010b,a) the inferred fluctuation anisotropy is found to be only $k_{\perp} / k_{\|} \sim 3$. This ratio is significantly smaller than the anisotropy ratio we expect for the low-frequency KAWs.

According to the turbulence theory of Howes et al. (2008), the KAWs are argued to play a major role in the local heating and acceleration of the solar wind protons. One of the consequence of their results is that the protons should receive thermal energy through Landau damping of the turbulent fluctuations near the proton gyroscale. However, since the dispersive KAWs require $\omega \ll \Omega_{\mathrm{p}}$, they can neither damp via resonant ion-cyclotron wave dissipation nor cause the observed (see the subsequent section) diffusion and resulting perpendicular heating of the protons.

Recent work by He et al. (2011) provided possible evidence of Alfvén/ion-cyclotron waves in the angle distribution of magnetic helicity of solar wind turbulence. Therefore, the fluctuations observed (Chen et al., 2010b,a) around and slightly above the proton inertial length scale are likely a combination of oblique Alfvén/ion-cyclotron and KAW/fastmode/whistler waves.

\section{Cyclotron-resonance condition and the dispersion re- lation of oblique magnetohydrodynamic waves}

The problem arises under which conditions the above discussed general plateaus of Eq. (9) are really accessible to the particles, and for which kind of waves other than parallel ion-cyclotron waves. Accessibility is a condition for obtaining effective diffusion in the considered wave field, a process which depends essentially on the particles' plasma beta, $\beta_{j}=\left(v_{j} / V_{\mathrm{A}}\right)^{2}$, since it determines the width of their distribution function in velocity space. But also the resonance condition (10) must be obeyed. The corresponding resonance speed (Eq. 8) usually is much larger than the thermal speed, i.e. $\left|V_{j}(\mathbf{k}, s)\right| \gg v_{j}$, and thus often there are no resonant particles among the thermal population. For example, for lowfrequency magnetohydrodynamic waves, such as Alfvén and fast-mode waves (with $\left|y_{\|}\right| \ll 0$ ), the resonances (see Eq. 10 again) lay out in the far tails of the distributions, where there is only an exponentially small number of particles. Only if $s=0$ Landau resonance is possible, or for $s= \pm 1$ and $s= \pm n$ with $n$ larger than unity, the first- and higher-order cyclotron resonances can occur, in which cases the parallel resonance speed may become located within the thermal domain of the distribution.

Let us first consider the cases of parallel propagating left-handed (LH, minus sign below in Eq. 21) Alfvén/ioncyclotron (AIC) mode waves and right-handed (RH, plus sign below in Eq. 21) fast-magnetoacoustic-whistler (FMW) mode waves. Their approximate cold-plasma dispersion relations give a phase speed that can be written as a function of frequency $\omega$ as

$C_{ \pm}(\omega)=V_{\mathrm{A}} \sqrt{1 \pm x}=V_{\mathrm{A}} \sqrt{1 \pm\left|y_{\|}\right| C_{ \pm}}$,

with $x=\omega / \Omega_{j}$. This equation can be solved for the positive phase speed as a function of the parallel wave number and yields

$\frac{C_{ \pm}\left(y_{\|}\right)}{V_{\mathrm{A}}}= \pm \frac{1}{2}\left|y_{\|}\right|+\sqrt{1+\left(\frac{1}{2} y_{\|}\right)^{2}}$.

This implies the well known result that the AIC wave slows down to zero phase speed for large wave number $\left(y_{\|} \rightarrow\right.$ $\infty)$, whereas the FMW wave essentially propagates at the Alfvén speed and then faster, where at higher wave number the whistler dispersion relation, $x=y_{\|}^{2}$, is attained. Thus whereas parallel AIC wave can resonate with the bulk ions, the parallel FMW wave can according to Eq. (10) merely resonate with the tail but not core of an ion distribution. Consequently, to move that cyclotron resonance into the thermal part of an ion distribution function requires oblique wave propagation.

Turning now to magnetoacoustic waves, we first note that to fulfill the resonance requirement (Eq. 10), one must consider genuine high-frequency waves, which is to say shortwavelength waves for which $k \approx\left|\Omega_{j}\right| / V_{\mathrm{A}}$. Therefore, we 
will consider oblique slow and fast mode waves (or the whistler wave), for which the parallel dispersion relation was already given by the $C_{+}\left(y_{\|}\right)$of Eq. (21), and for which the resulting phase speed varies, for $0<\left|y_{\|}\right|<1$, between one to two times $V_{\mathrm{A}}$. For oblique propagation and small plasma betas, the phase speed is of similar size than that for parallel propagation. As the dispersion of the fast-mode wave remains still weak around the proton gyrofrequency, we shall for the moment neglect dispersion entirely and consider (according to our definition in Eq. 8) the phase speed named $C_{\mathrm{F}, \mathrm{S}}$ resulting from magnetohydrodynamics (Melrose and McPhedran, 1991) for the fast and slow magnetoacoustic waves. This speed only depends on the wave propagation angle $\theta$ as follows:

$$
\begin{aligned}
& C_{\mathrm{F}, \mathrm{S}}(\theta)=\frac{\omega_{\mathrm{F}, \mathrm{S}}(\mathbf{k})}{k_{\|}}=\sqrt{\left(1+\tan ^{2} \theta\right)} \times \\
& \quad \sqrt{\frac{1}{2}\left(C_{\mathrm{MS}}^{2} \pm\left(\left[V_{\mathrm{A}}^{2}-\bar{C}_{\mathrm{S}}^{2}\right]^{2}+4 V_{\mathrm{A}}^{2} \bar{C}_{\mathrm{S}}^{2} \sin ^{2} \theta\right)^{1 / 2}\right)},
\end{aligned}
$$

with the magnetosonic speed $C_{\mathrm{MS}}=\sqrt{V_{\mathrm{A}}^{2}+\bar{C}_{\mathrm{S}}^{2}}$, the sound speed $\bar{C}_{\mathrm{S}}$, and $\tan \theta=y_{\perp} / y_{\|}=k_{\perp} / k_{\|}$. For low beta, with the definition $\beta=\left(\bar{C}_{\mathrm{S}} / V_{\mathrm{A}}\right)^{2}$, the fast mode will essentially travel at the Alfvén speed in all directions, and thus the parallel phase speed $C_{\mathrm{F}}(\theta)$ will with increasing angle grow strongly beyond $V_{\mathrm{A}}$ for oblique propagation. In contrast, the slowmode parallel phase speed $C_{\mathrm{S}}(\theta)$ will not change a lot with $\theta$ but essentially stay at the sound speed $\bar{C}_{\mathrm{S}}$, and similarly the parallel Alfvén phase speed simply is constant and given by $C_{\mathrm{A}}(\theta)=V_{\mathrm{A}}$. The resonance condition for Alfvén waves (with $\omega_{\mathrm{A}}=V_{\mathrm{A}} k_{\|}$) of any propagation direction then yields the resonant speed,

$V_{\|}\left(y_{\|}\right)=V_{\mathrm{A}}\left(1-\frac{s}{y_{\|}}\right)=\frac{V_{\mathrm{A}}}{y_{\|}}\left(\frac{\omega_{\mathrm{A}}}{\Omega_{j}}-s\right)$,

and for the non-dispersive fast and slow waves the resonant speed,

$V_{\|}\left(y_{\|}\right)=C_{\mathrm{F}, \mathrm{S}}\left(\theta\left(y_{\|}\right)\right)-V_{\mathrm{A}} \frac{s}{y_{\|}}=\frac{V_{\mathrm{A}}}{y_{\|}}\left(\frac{\omega_{\mathrm{F}, \mathrm{S}}}{\Omega_{j}}-s\right)$,

with $s= \pm 1$ being the values for lowest-order cyclotron resonances. The angle is a function of $y_{\|}$via the relation: $\theta=\arctan \left(y_{\perp} / y_{\|}\right)$. Therefore, in both cases the speed $V_{\|}$ can attain any value between $+\infty$ and $-\infty$, if $y_{\|}$is allowed to vary in the same parameter range. Thus for oblique propagation (i.e. when both $s=1$ and $s=-1$ have to be considered) the full resonance circle, given for instance by Eq. (13) for Alfvén waves, becomes accessible to the ions. Dispersion makes the situation more complicated and will not be considered here.

For sake of simplicity, we may now neglect the $y_{\|}$dependence of $\theta$ in $C_{\mathrm{F}, \mathrm{S}}$ in Eq. (22), and can then integrate Eq. (12) directly over $y_{\|}$. Like in the previous section, the simple result is $I=V_{\|} C_{\mathrm{F}, \mathrm{S}}$, and thus up to an unimportant constant the specific particle energy in the wave frame determines the resonance plateaus, which are circles with a $\theta$-dependent radius, and again given by the specific energy:

$E_{\mathrm{F}, \mathrm{S}}\left(V_{\|}, V_{\perp}, \theta\right)=\frac{1}{2}\left(V_{\perp}^{2}+\left(V_{\|}-C_{\mathrm{F}, \mathrm{S}}(\theta)\right)^{2}\right)$.

We will use this equation in the subsequent section and apply it to measured ion velocity distribution functions. Considering moderately oblique, i.e. quasi-parallel propagation $0<\theta<45^{\circ}$, and moderately short parallel wavelength, i.e. $y_{\|}<1$, then the fast mode speed $C_{\mathrm{F}}$ is still close to $V_{\mathrm{A}}$. According to Eq. (13) for parallel and oblique Alfvén waves and Eq. (25) for fast waves, particles will mostly diffuse in circles centered on the Alfvén velocity. Considering only antisunward (with $y_{\|}>0$ ) and oblique wave propagation permits to use Eqs. (23) and (24) to define the $V_{\|}$range, which for $s=1$ is: $-\infty<V_{\|} \leq 0$; and for $s=-1$ it is: $0 \leq V_{\|}<\infty$, whereby $y_{\|}$is assumed to vary between zero and infinity. If we have sunward waves as well, the reversed situation occurs, however the in-situ wave and turbulence observations (Tu and Marsch, 1995) indicate predominant outward propagation of fluctuations of solar origin, which will cause an asymmetry in the pitch-angle scattering of the particles in the turbulent wave field. Using Eqs. (21) and (24), one can determine for $s=1$ the critical wavenumber $y_{\| 0}$ for the FMW wave, at which the parallel speed $V_{\|}$vanishes. One finds

$$
\frac{1}{y_{\| 0}}=\frac{1}{2} y_{\| 0}+\sqrt{1+\left(\frac{1}{2} y_{\| 0}\right)^{2}} \text {, }
$$

which yields $y_{\| 0} \approx 0.7$ as numerical solution. This solution is relevant for oblique propagation (i.e. $y_{\perp} \neq 0$ ) as well, which permits one to consider also the case $s=1$ for the FMW wave, in addition to the case $s=-1$, which solely is allowed for parallel propagation. After these theoretical preparations we are ready to investigate the measured VDFs with respect to possible signatures for resonant diffusion in waves.

\section{Diffusion plateaus in observed velocity distributions}

Ten years ago, Marsch and Tu (2001a) found the first clear observational evidence in the Helios ion velocity distribution functions (VDFs) for the occurrence of diffusion-related plateaus formed by the solar wind protons. The core of their VFDs appeared to be in resonance predominantly with outward (but partly also with inward) propagating left-hand polarized parallel ion cyclotron waves, and by wave-induced pitch-angle scattering attain a bi-shell shape. The anisotropy regulation and plateau formation through resonant pitchangle diffusion of solar wind protons was further studied by Tu and Marsch (2002). Then Heuer and Marsch (2007) corroborated these results on the basis of Helios data analysis with a large statistical ensemble, encompassing many typical proton VDFs in fast solar wind streams as measured by Helios at various heliocentric distances. Particle-in-cell 
simulations of Alfvén/ion-cyclotron wave scattering by Gary and Saito (2003) had previously also demonstrated the ability and effectiveness of this process in shaping proton VDFs. Later, Marsch et al. (2004) studied in detail the temperature anisotropy of the core part of the proton VDFs in association with some related kinetic instabilities, and Marsch et al. (2006) analysed the limits on the proton anisotropy as defined by the fluid fire-hose and mirror instabilities for the Helios data.

The above mentioned studies concentrated on the core part of the VDFs of the protons, yet it had been known for some time, see, e.g. Gary (1991), that a proton beam may interact resonantly with right-hand polarized parallel fast magnetosonic waves, a process which can slow down and broaden the beam observed in solar wind proton VDFs, and thus regulate its drift speed (Dum et al., 1980; Marsch, 1991; Daughton and Gary, 1998; Tu et al., 2002). Here we shall also consider the proton beam and, by use of the theoretical deliberations of the previous sections, analyse the effects of pitch-angle scattering within the full range for $V_{\|}$(from minus to plus infinity). Our theoretical scope includes obliquely propagating MHD waves and extends their dispersion relation into the kinetic domain, but we will consider only the simplified wave phase speed $C(\boldsymbol{k})$ of Eq. (22) without any dispersive corrections. Note that in the following figures the plasma $\beta$ is low (ranging between 0.1 and 0.25 ), and thus $C_{\mathrm{F}}(\theta) \approx V_{\mathrm{A}} \sqrt{\left(1+\tan ^{2} \theta\right)}$, which for quasi-parallel $\left(<45^{\circ}\right)$ propagation is essentially equal to the Alfvén speed $V_{\mathrm{A}}$.

The subsequent Helios data (taken on day 105 of the year 1976) analysis will focus on some of the very nonthermal VDFs which are typical for the fast solar wind near the Helios perihelion at 0.3 AU, which reveals (Marsch et al., 1982) the most pronounced proton core temperature anisotropies and fastest and hottest beams. The data analysis procedures and methods to construct the distribution have been amply described in the above references and need not be repeated here. The VDFs shown below are not contaminated by alpha particles, which were simultaneously measured by the Helios plasma experiment (Rosenbauer et al., 1977) but could reliably be separated from the protons in the VDFs presented subsequently.

The VDFs in the following figures are plotted as isodensity contours (as continuous lines) at values of $80,60,40$, and $20 \%$ of the maximum value of a given VDF and then (as dotted lines) at values of 10, 3.3, 1, 0.33, 0.1\%. The cuts through the three-dimensional VDFs are provided in planes defined by the proton bulk velocities ( $V_{\mathrm{x}}$-axis, roughly corresponding to the radial direction) and the local magnetic field vector. The $V_{\mathrm{y}}$-axis is just orthogonal to the $V_{\mathrm{x}}$-axis in that plane. The field direction is along the line connecting the maximum of a VDF with the Alfvén point (in the tail) indicated respectively by a fat dot.

The contours were obtained by interpolation of the VDF between the actually measured points in phase space. Some of these contours appear to be abruptly cut by wavy lines, which is an artifact due to the limited angular resolution of the plasma instrument which cannot always cover the VDF fully. Superposed on the phase space density are concentric circles, which all have a common center that is the position of the Alfvén velocity, corresponding to a point located away from the maximum on the straight thick line that represents the local magnetic field direction and axis of gyro-symmetry. The spacing of those circles has been chosen to match the spacing of the measured contour lines at the sunward (negative $V_{\|}$) side of the velocity plane.

The four VFDs displayed in Fig. 1 in panels (a)-(d) reveal the striking characteristics of diffusion, which has the effect that the isocontours follow the concentric circles prescribed by proton pitch-angle scattering in weakly dispersive waves that propagate along the mean field with a parallel phase speed $C(\boldsymbol{k})=V_{\mathrm{A}}$, like parallel or oblique Alfvénic fluctuations do. In particular their core parts are not simply elliptically shaped but slightly bent, such that the contours are smoothly nested to the curvature and embedded in the bending of the larger circles. This matching of the core has been described before and analysed in detail in the papers of Marsch and Tu (2001a); Tu and Marsch (2002); Heuer and Marsch (2007), in which parallel wave propagation was assumed. In contrast, here we will also allow for oblique propagation of Alfvén and slow-mode waves and consider their scattering effects.

At this point it is important to mention that diffusive particle scattering by waves may already take place in the inner solar wind and outer corona, where major spectral characteristics of radio scattering and scintillation inferred from the density structure function has lead Harmon and Coles (2005) to conclude from their radio measurements that "The highfrequency flattening of the plasma density fluctuation spectrum can be explained simply by the enhanced compressibility of the linear, obliquely propagating Alfvén wave in the ion cyclotron regime".

Note that diffusion, which is due to weakly dispersive Alfvén/ion-cyclotron waves propagating downstream away from the Sun, can only affect protons with negative $V_{\|}$. Protons in the core with positive $V_{\|}$require upstream waves which cannot have come from the Sun directly but are generated locally. The work by Jian et al. (2010) has clearly shown their existence in the inner heliosphere at $0.3 \mathrm{AU}$ from data of the Messenger mission, and near Earth orbit at $1 \mathrm{AU}$ from STEREO data (Jian et al., 2009). The paper by Bourouaine et al. (2010) has revealed the close correlation of the waves with the proton core temperature anisotropy in the Helios data. According to Heuer and Marsch (2007) it is clear that dispersive upstream waves are needed to shape the positive-speed half of the proton core distribution. Yet to study this is not the aim of the present paper. Here we will concentrate on the extended bulge and beam occurring at larger speeds, even beyond the Alfvén speed (located at the center of the circular ring system). 

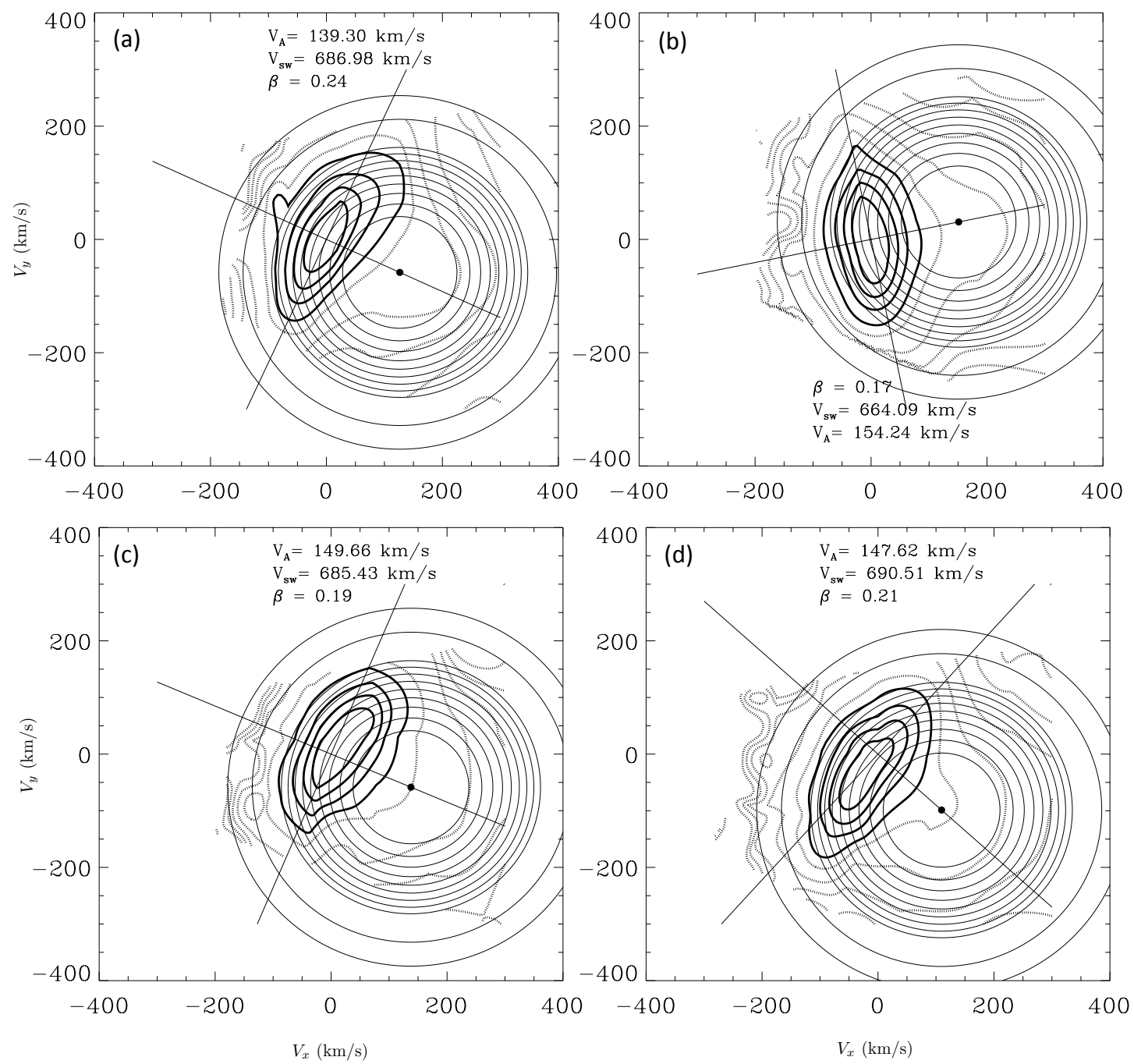

Fig. 1. Proton velocity distribution functions in fast solar wind, with the corresponding Alfvén speed and wind speed indicated, together with the plasma beta. Dark continuous isodensity contours relate to 80,60, 40, and $20 \%$ of the maximum, and the respective dotted contours to $10,3.3,1,0.33$, and $0.1 \%$. The local magnetic field is the symmetry axis for these gyrotropic VDFs. The transverse straight line in the perpendiuclar direction to the field corresponds to a parallel speed of $V_{\|}=0$, which defines the solar wind frame centered about in the maximum of the core protons. The location of the Alfvén speed is indicated by a dark dot, which is the center of the circular diffusion lines along which protons pitch-angle scatter in oblique Alfvén waves. The spacings of the concentric circles are determined by the variable spacings (radii) of the measured contour lines, and given by their intersections with the negative and positive $V_{\|}$axis, i.e. the magnetic field direction. The abrupt cutoffs of some contours are artifacts induced by the finite resolution of the plasma instrument in velocity space. The measurements of the VDFs were made on 14 April 1976 at the times (hours:minutes:seconds): (a) 22:43:08, (b) 23:07:26, (c) 23:20:56, and (d) 23:42:32. All data correspond to a heliocentric distance of $0.3 \mathrm{AU}$.

Close inspection of the four panels (a)-(d) of Fig. 1 shows that the measured (dotted lines) contours near and below the $10 \%$ level largely coincide, at the positive speeds beyond the Alfvén speed, with the circles, and in fact are nicely fitted by them. A note of caution is in order, though, that the dotted contours might be less reliable statistically, as the corresponding counts in the plasma detector are comparatively low, yet still above the critical one-count level. However, a fortuitous coincidence seems unlikely, as the trends appearing in the comparison between measured contours and predicted plateaus are systematic and lasting. For example, in panel (a) the beam or heat-flux carrying tail of the proton VDF is formed by the widely spaced contours encircling the local Alfvén speed of $155 \mathrm{~km} \mathrm{~s}^{-1}$. Similarly, in the panels (b)-(d) the proton tail is very broad and appears to be formed by the wide pitch-angle scattering contours. This broad tail has a much higher intrinsic temperature (kinetically defined by the limited partial second moment with respect to the wave frame of reference) than the cooler core part. Furthermore, the kinematics of pitch-angle scattering 

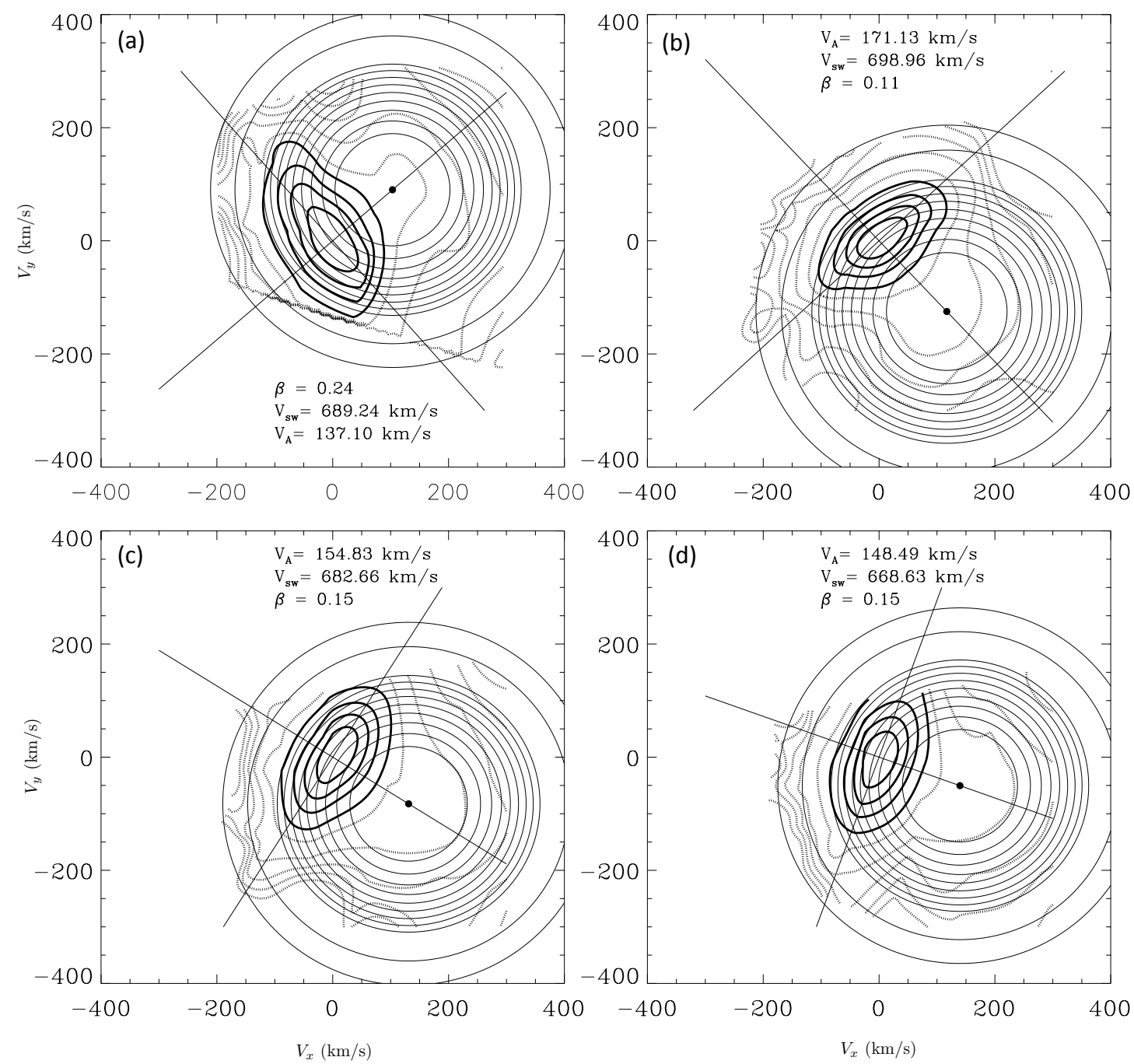

Fig. 2. Proton velocity distribution functions in fast solar wind, presented in the same format as in the previous figure. Note, however, the stronger and more pronounced beams and their higher temperatures, which are apparent by the wide almost circular bulges. The measurements of the VDFs were made on 14 April 1976 at the times (hours:minutes:seconds): (a) 20:38:13, (b) 22:02:35, (c) 23:09:27, and (d) 23:10:08. All data correspond to a heliocentric distance of 0.3 AU.

automatically enhances the magnetic moment of a proton, and as a net result this mechanism produces a temperature anisotropy with $T_{\perp}>T_{\|}$.

In Fig. 2 we present four other examples of proton VDFs, now with a more distinct field-aligned tail or beam and a less bent core component. Whereas the proton beam is broad and roundish in the cases (c)-(d), where the circles closely embrace the beam plateau, the cases (a)-(b) reveal a significant enhancement along the magnetic field, which corresponds to a narrower beam of the kind predicted by simulation of the parametric decay of Alfvén waves according to Araneda et al. (2008) and Araneda et al. (2009). Such a distinct proton tail may develop due to Landau damping by acoustic daughter waves (or preexisting slow mode waves) on the core protons with $V_{\|}>0$. But also here the cyclotron damping of protons, undergoing pitch-angle scattering at speed $V_{\|}>V_{\mathrm{A}}$ by Alfvén/ion-cyclotron daughter waves, is quite obvious in the perpendicularly stretched contours.

Such perpendicular heating of the beam is quite common and was in fact early noticed in the ion data by the Helios plasma experiment team (Rosenbauer et al., 1981), who already suggested to explain the nonthermal proton features in terms of wave-particle interactions. Here we corroborate this early finding quantitatively, but moreover interpret it convincingly by quasi-linear pitch-angle scattering.

It should be noted that the observed VDFs can, at their negative-speed halves, well be interpreted if the diffusion of the protons (particularly of those that already have a substantial magnetic moment) is caused by dispersion-less parallel Alfvén waves. This is obvious in all of the VDFs shown 

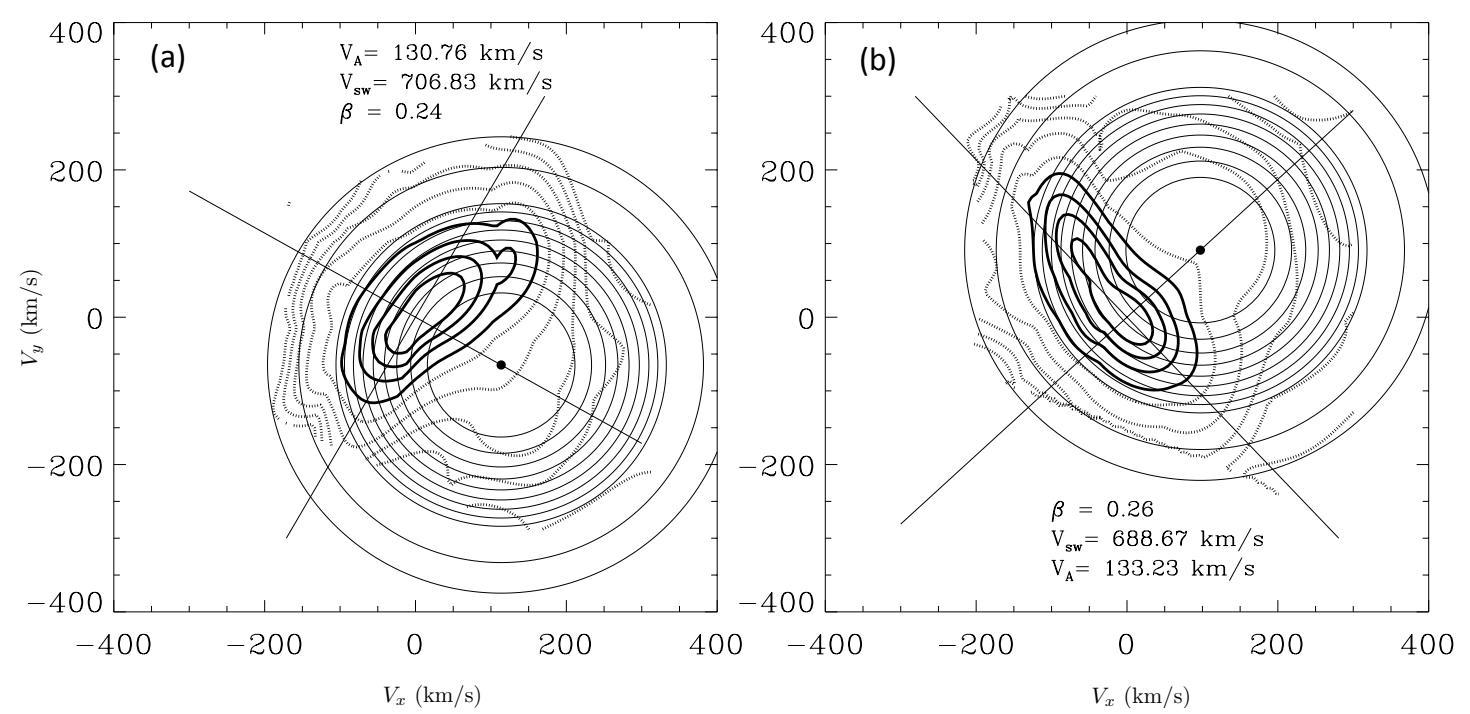

Fig. 3. Proton velocity distribution functions in fast solar wind, presented in the same format as in the previous two figures. Close inspection shows, however, the more strongly deformed cores (continuous contours), which are bent around and nestled to the circular diffusion lines and reach out to the positive half of velocity space. The measurements of the VDFs were made on 14 April 1976 at the times (hours:minutes:seconds): (a) 21:59:53 and (b) 22:24:14. The data correspond to a heliocentric distance of 0.3 AU.

in Figs. 1 and 2. Moreover, these low-frequency waves when being oblique can also resonate with protons at positive speeds according to Eq. (23). As mentioned before, the fast-mode dispersion branch extends in frequency well above the proton cyclotron frequency. Thus the oblique fast mode waves can have much higher phase speeds than the Alfvén speed, and therefore after Eq. (24) oblique fast waves can also resonate with protons at positive $V_{\|}$.

However, a system of concentric circles surrounding the corresponding phase speed only fits the measurements well if the associated fast-mode phase speed remains close to the Alfvén speed (see again Eq. 21). This is of course ensured for waves with $k<\Omega_{p} / V_{\mathrm{A}}$, i.e. if $y_{\| 0}<0.7$ according to Eq. (26), which means for waves belonging still to the MHD turbulence domain in the solar wind (Tu and Marsch, 1995). Similarly, the KAWs which we discussed extensively in the previous sections will normally also have a phase speed which is clearly above the local Alfvén speed (see again Eq. 14 for $y_{\perp}>1$ ). So, this wave mode also is unlikely to shape the VDFs of solar wind protons, however it may have an effect on the suprathermal electrons.

In conclusion, oblique wave propagation is essential in enabling the protons to diffuse across the line $V_{\|}=0$, i.e. from negative for $s=1$ to positive for $s=-1$ parallel resonant speed according to Eq. (23), for the oblique left-handed Alfvén/ion-cyclotron wave. Similarly, this transition across the line $V_{\|}=0$ is enabled by an oblique fast-mode wave, i.e. from negative (for $s=1$ ) to positive (for $s=-1$ ) parallel resonant speed according to Eq. (23), assuming that the wave frequencies $\omega_{\mathrm{A}}$ and $\omega_{\mathrm{F}}$ are positive and smaller than $\Omega_{\mathrm{p}}$, consistent with the phase speed being near the Alfvén speed.
Two exemplary VDFs, which clearly indicate that such diffusion from negative to positive resonant speeds does occur, are shown in Fig. 3, with both panels (a)-(b) being set up in the same format as the ones shown before. Here the perpendicular (in the direction of the temperature anisotropy) line indicates the location of $V_{\|}=0$, which about coincides with the position of the maxima of the VDFs. Note that the dotted contours are clearly bent around and largely follow the circular plateau lines, which demonstrates that the diffusion process does not stop at $V_{\|}=0$ but goes well beyond that border. This is even more remarkable as the measured contours are sparsely interpolated but their derived curvatures are fairly close to being circular. We recall that dispersion of the waves (most relevant in the core) would lead to other shapes of the VDFs, as was shown previously by Heuer and Marsch (2007). Apparently, wave dispersion effects are of minor importance in the tails of the distributions (given by the dotted contours). However, for this to be the case oblique propagation of the waves at work is essential, because only then can diffusion occur over wide segments of the plateau circles that are centered at the Alfvén speed.

\section{Conclusions}

We have analysed the effects that wave diffusion can have on the shape of the proton velocity distribution functions in the fast solar wind. The basic predictions of quasilinear theory for wave-particle interactions have been largely validated through a detailed comparison with the in-situ plasma measurements made by the Helios spacecraft. The results obtained agree favourably with the physical picture 
of resonant diffusion of the protons in obliquely propagating Alfvén/ion-cyclotron waves below and fast magneto-acoustic waves around the proton cyclotron frequency.

As the measured contours not always fully match the simple circular plateaus for non-dispersive waves, we may conclude that the wave-scattering of the protons is partly inelastic and thus associated with weak absorption or emission of the waves involved, processes which are important for the dissipation of magnetohydrodynamic turbulence in the fast solar wind where Alfvénic fluctuations are the prevailing component. Also, dispersion effects may be important even in the tails of the VDFs, but certainly they matter in the core of the VDFs as was shown in our previous work cited above. Like it, the present study showed that the solar wind plasma while carrying large-amplitude turbulent fluctuations cannot remain Maxwellian. In a self consistent way the proton diffusion in the wave field strongly distorts the VDFs and gives them their observed nonthermal shape, typically with a bi-shell anisotropic core and substantial beam along the magnetic field.

Acknowledgements. The service charges for this open access publication have been covered by the Max Planck Society.

Topical Editor R. Forsyth thanks one anonymous referee for her/his help in evaluating this paper.

\section{References}

Araneda, J. A., Marsch, E., and Viñas, A. F.: Proton Core Heating and Beam Formation via Parametrically Unstable Alfvén-Cyclotron Waves, Phys. Rev. Lett., 100, 125003, doi:10.1103/PhysRevLett.100.125003, 2008.

Araneda, J. A., Maneva, Y., and Marsch, E.: Preferential Heating and Acceleration of $\alpha$ Particles by Alfvén-Cyclotron Waves, Phys. Rev. Lett., 102, 175001, doi:10.1103/PhysRevLett.102.175001, 2009.

Bourouaine, S., Marsch, E., and Neubauer, F. M.: Correlations between the proton temperature anisotropy and transverse highfrequency waves in the solar wind, Geophys. Res. Lett., 37, L14104, doi:10.1029/2010GL043697, 2010.

Brambilla, M.: Kinetic Theory of Plasma Waves, Homogeneous Plasmas, Clarendon Press, Oxford, 1998.

Chen, C. H. K., Horbury, T. S., Schekochihin, A. A., Wicks, R. T., Alexandrova, O., and Mitchell, J.: Anisotropy of Solar Wind Turbulence between Ion and Electron Scales, Phys. Rev. Lett., 104, 255002, doi:10.1103/PhysRevLett.104.255002, 2010a.

Chen, C. H. K., Wicks, R. T., Horbury, T. S., and Schekochihin, A. A.: Interpreting power anisotropy measurements in plasma turbulence, Ap. J., 711, L79, doi:10.1088/2041-8205/711/2/L79, 2010b.

Daughton, W. and Gary, S. P.: Electromagnetic proton/proton instabilities in the solar wind, J. Geophys. Res., 103, 20613-20620, doi:10.1029/98JA01385, 1998.

Dum, C. T., Marsch, E., and Pilipp, W. G.: Determination of wave growth from measured distribution functions and transport theory, J. Plasma Phys., 23, 91-113, 1980.
Gary, S. P.: Electromagnetic ion/ion instabilities and their consequences in space plasmas: A review, Space Sci. Rev., 56, 373$415,1991$.

Gary, S. P. and Saito, S.: Particle-in-cell simulations of Alfvéncyclotron wave scattering: Proton velocity distributions, J. Geophys. Res., 108, 1194, doi:10.1029/2002JA009824, 2003.

Harmon, J. K. and Coles, W. A.: Modelling radio scattering and scintillation observations on the inner solar wind using oblique Alfvén/ion cyclotron waves, J. Geophys. Res., 110, A03101, doi:10.1029/2004JA010834, 2005.

He, J.-S., Marsch, E., Tu, C.-Y., Yao, S., and Tian, H.: Possible evidence of Alfvén-cyclotron waves in the angle distribution of magnetic helicity of solar wind turbulence, Astrophys. J., 731:85, doi:10.1088/0004-637X/731/2/85, 2011.

Heuer, M. and Marsch, E.: Diffusion plateaus in the velocity distributions of fast solar wind protons, J. Geophys. Res., 112, A03102, doi:10.1029JA011979, 2007.

Hollweg, J. V.: Kinetic Alfvén wave revisited, J. Geophys. Res., 104, 14811-14820, 1999.

Howes, G. G., Cowley, S. C., Dorland, W., Hammett, G. W., Quataert, E., and Schekochihin, A. A.: A model of turbulence in magnetized plasmas: Implications for the dissipation range in the solar wind, J. Geophys. Res., 113, 5103, doi:10.1029/2007JA012665, 2008.

Howes, G. G., Bale, S. D., Klein, K. G., Chen, C. H. K., Salem, C. S., and TenBarge, J. M.: The slow-mode nature of compressible wave power in solar wind turbulence, arXiv:1106.4327v1 [astroph.SR], 2011.

Isenberg, P. A. and Lee, M. A.: A dispersive analysis of bispherical pickup ion distributions, J. Geophys. Res., 101, 11055-11066, 1996.

Jian, L. K., Russell, C. T., Luhmann, J. G., Strangeway, R. J., Leisner, J. S., and Galvin, A. B.: Ion cyclotron waves in the solar wind observed by STEREO near 1 AU, Astrophys. J., 701, L105, doi:10.1088/0004-637X/701/2/L105, 2009.

Jian, L. K., Russell, C. T., Luhmann, J. G., Anderson, B. J., Boardsen, S. A., Strangeway, R. J., Cowee, M. M., and Wennmacher, A.: Observations of ion cyclotron waves in the solar wind near 0.3 AU, J. Geophys. Res., 115, A12115, doi:10.1029/2010JA015737, 2010.

Kellogg, P. J. and Horbury, T. S.: Rapid density fluctuations in the solar wind, Ann. Geophys., 23, 3765-3773, doi:10.5194/angeo23-3765-2005, 2005.

Kennel, C. F. and Engelmann, F.: Velocity space diffusion from weak plasma turbulence in a magnetic field, Phys. Fluids, 9, 2377-2388, 1966.

Mann, G., Hackenberg, P., and Marsch, E.: Linear mode analysis in multi-ion plasmas, J. Plasma Phys., 58, 205-221, 1997.

Marsch, E.: Kinetic Physics of the Solar Wind Plasma, in: Physics of the Inner Heliosphere, Vol. 2: Particles, Waves and Turbulence, edited by: Schwenn, R. and Marsch, E., vol. 20 of Physics and Chemistry in Space, 45, Springer, New York, 1991.

Marsch, E.: On resonant interactions of ions with plasma waves in a reduced quasi-linear theory, Nonlin. Processes Geophys., 9, 69-74, doi:10.5194/npg-9-69-2002, 2002.

Marsch, E.: Kinetic Physics of the Solar Corona and Solar Wind, Living Rev. Solar Phys., 3, available at: http://www. livingreviews.org/lrsp-2006-1, 2006.

Marsch, E. and Tu, C.-Y.: Correlations between the fluctuations 
of pressure, density, temperature and magnetic field in the solar wind, Ann. Geophys., 11, 659-677, 1993.

Marsch, E. and Tu, C. Y.: Evidence for pitch-angle diffusion of solar wind protons in resonance with ion-cyclotron waves, J. Geophys. Res., 106, 8357-8361, 2001a.

Marsch, E. and Tu, C. Y.: Heating and acceleration of coronal ions interacting with plasma waves through cyclotron and Landau resonance, J. Geophys. Res., 106, 227-238, 2001b.

Marsch, E., Mühlhäuser, K.-H., Schwenn, R., Rosenbauer, H., Pilipp, W. G., and Neubauer, F. M.: Solar wind protons: Threedimensional velocity distributions and derived plasma parameters measured between 0.3 and 1 AU, J. Geophys. Res., 87, 5272, 1982.

Marsch, E., Ao, X.-Z., and Tu, C.-Y.: On the temperature anisotropy of the core part of the proton velocity distribution function in the solar wind, J. Geophys. Res., 109, A04102, doi:10.1029/2003JA010330, 2004.

Marsch, E., Zhao, L., and Tu, C.-Y.: Limits on the core temperature anisotropy of solar wind protons, Ann. Geophys., 24, 20572063, doi:10.5194/angeo-24-2057-2006, 2006.

Matthaeus, W. H., Klein, L. W., Ghosh, S., and Brown, M. R.: Nearly incompressible magnetohydrodynamics, pseudosound, and solar wind fluctuations, J. Geophys. Res., 96, 5421-5435, 1991.

Melrose, D. B. and McPhedran, R. C.: Electromagnetic Processes in Dispersive Media, Cambridge Univiversity Press, New York, 1991.

Rosenbauer, H., Schwenn, R., Marsch, E., Meyer, B., Miggenrieder, H., Montgomery, M. D., Mühlhäuser, K. H., Pilipp, W., Voges, W., and Zink, S. M.: A survey on initial results of the HELIOS plasma experiment, J. Geophys., 420, 561-580, 1977.
Rosenbauer, H., Marsch, E., Mühlhäuser, K. H., Pilipp, W., and Schwenn, R.: Indications of resonant wave-particle interactions in fast stream solar wind distributions, in: Solar Wind Four, edited by: Rosenbauer, H., Report No. MPAE-W-100-81-31, Max-Planck-Institut für Aeronomie, Katlenburg-Lindau, Germany, pp. 353-358, 1981.

Stix, T. H.: Waves in Plasmas, American Institute of Physics, New York, 1992.

Tu, C.-Y. and Marsch, E.: On the nature of compressive fluctuations in the solar wind, J. Geophys. Res., 99, 21481-21509, 1994.

Tu, C.-Y. and Marsch, E.: MHD structures, waves and turbulence in the solar wind: Obervations and theories, Space Sci. Rev., 73, 1-210, 1995.

Tu, C.-Y. and Marsch, E.: Anisotropy regulation and plateau formation through pitch angle diffusion of solar wind protons in resonance with cyclotron waves, J. Geophys. Res., 107, 1249, doi:10.1029/2001JA000150, 2002.

Tu, C.-Y., Marsch, E., and Qin, Z.-R.: Dependence of the proton beam drift velocity on the proton core plasma beta in the solar wind, J. Geophys. Res., 109, 101, doi:10.1029/2004JA010391, 2004.

Yao, S., He, J.-S., Marsch, E., Tu, C.-Y., Pedersen, A., Rème, H., and Trotignon, J. G.: Multi-scale Anti-correlation Between Electron Density and Magnetic Field Strength in the Solar Wind, Ap. J., 728, 146, doi:10.1088/0004-637X/728/2/146, 2011. 